\title{
Efecto del color de la cubierta seminal sobre la imbibición, la germinación y la estructura de la semilla de Crotalaria juncea (Fabaceae)
}

\author{
Effect of Seed Coat Color on Imbibition, Germination and Seed \\ Structure of Crotalaria juncea [Fabaceae]
}

\author{
Beltramini, Verónica S.; Ana L. Pascualides* \\ Botánica Morfológica, Facultad de Ciencias Agropecuarias. Avda. Valparaíso y Félix Aldo Marrone 746 , \\ Ciudad universitaria, Universidad Nacional de Córdoba, (5000) Córdoba, Argentina. \\ * Autor corresponsal: apascual@agro.unc.edu.ar
}

\begin{abstract}
Resumen - Beltramini, Verónica S.; Ana L. Pascualides. 2017. "Efecto del color de la cubierta seminal sobre la imbibición, la germinación y la estructura de la semilla de Crotalaria juncea (Fabaceae)". Lilloa 54 (2). Crotalaria juncea L. es una leguminosa subtropical herbácea, nativa de India y Paquistán. Las semillas presentan policromía en la cubierta seminal. Los estudios sobre el color del tegumento seminal que relacionen la estructura interna de la semilla de C. juncea, con la capacidad de imbibición y germinación son escasos. El objetivo de este trabajo fue estudiar el efecto de los colores de la cubierta seminal sobre la imbibición, la germinación y los componentes de la semilla. Se evaluaron la proporción de cada componente de la semilla (cubierta seminal, endosperma y embrión), y la cantidad de agua embebida por cada uno de ellos, el espesor de la cubierta seminal y del endosperma, el porcentaje de germinación y de emergencia de las semillas uniformes y variegadas. No existieron diferencias significativas en las proporciones de la cubierta seminal, el endosperma y el embrión entre los dos colores de tegumento seminal. Las semillas variegadas presentaron mayor porcentaje de agua en la cubierta seminal, las uniformes en el endosperma; el porcentaje de agua que retuvo el embrión fue similar en ambos grupos de semillas. Los resultados permiten afirmar que las semillas uniformes presentaron ventaja sobre las variegadas ya que el mayor porcentaje de agua lo absorbió el endosperma, cuya capa de aleurona fue de mayor espesor. Esto favorecería la hidratación del embrión para el inicio de la germinación. En las semillas variegadas el mayor porcentaje de agua se encontró en el episperma, donde las capas de macroesclereidas y parénquima tuvieron mayor espesor que en las semillas uniformes. Las semillas uniformes mostraron un alto porcentaje de germinación en el laboratorio y un mejor comportamiento en el campo que las variegadas.
\end{abstract}

Palabras clave: Episperma, endosperma, embrión, germinación, imbibición.

> Abstract - Beltramini, Verónica S.; Ana L. Pascualides. 2017. "Effect of seed coat color on imbibition, germination and seed structure of Crotalaria juncea. (Fabaceae)". Lilloa 54 (2). Crotalaria juncea L. is a subtropical herbaceous legume native of India and Pakistan. The seeds have polychromy in the seed coat. Studies on seed coat color that link the internal structure of Crotalaria juncea seed with imbibition and germination capacity are scarce. The aim of this work was to study the effect of seed coat colors on imbibition, germination, and seed components. The proportion of each component of the seed (seed coat, endosperm and embryo] and the amount of water absorbed for each one of them, the thickness of the seed coat and endosperm, the percentage of germination and emergence in the field of uniform and variegated seeds were evaluated. There were no significant differences in the proportions of the seed coat, endosperm and embryo between the two seed coat colors. Variegated seeds retained a higher percentage of water in the seed coat while uniform ones did it in the endosperm. The percentage of water retained the embryo was similar in both seed groups. Uniform seeds presented an advantage over variegated ones because in the first the highest

Recibido: 04/10/17 - Aceptado: 24/11/17 
percentage of water is absorbed by the endosperm, whose aleurone layer was thicker, which would promote embryo hydration for the start of germination. In variegated seeds, the highest percentage of water was found in the seed coat, where macrosclereids and parenchyma layers were thicker than in uniform seeds. The uniform seeds showed a high percentage of germination in the laboratory and a better behavior in the field that the variegated ones.

Keywords: Episperm, endosperm, embryo, germination, imbibition.

\section{INTRODUCCIÓN}

Crotalaria juncea L. es una leguminosa conocida como «suun hemp» o «crotalaria», nativa de India y Paquistán. Se cultiva en las regiones tropicales y subtropicales para abono verde y como fuente de fibra para la manufactura de sogas y papel de alta calidad (Cook y White, 1996). En el género Crotalaria se encontró una estrecha relación entre el color de la cubierta seminal o episperma y la permeabilidad (Saha y Takahashi, 1981; Buth y Narayan, 1987; Carreras et al., 2001). La cubierta seminal de las semillas de C. juncea presentó un patrón estable de tejidos (Miller, 1967; Pascualides y Planchuelo, 2007), y se caracterizó por ser permeable al agua (Pascualides y Planchuelo, 2007; ISTA, 2012). Sin embargo, el proceso de germinación depende no solo de la permeabilidad de la cubierta seminal, regulador de las interacciones entre la estructura de la semilla y el ambiente externo, sino también, de la elasticidad y la naturaleza del conjunto de tejidos que rodean al embrión (Egley, 1979; Manning y Van Staden, 1987; Mc Donald et al., 1988; Van Staden et al., 1989; Russi et al., 1992; Carreras et al., 2001; De Souza y Marcos-Filho 2001). La estructura de la semilla está ligada de manera intrincada a la función, sin embargo, son escasos los estudios que permiten esclarecer el modo en que los componentes de la semilla afectan el movimiento del agua en su interior.

Crotalaria juncea presentó semillas con cubierta seminal de distinta coloración, destacándose dos grupos principales, uno pardo-grisáceo oscuro y uniforme: semillas uniformes, y otro en la gama del castaño o con motas castañas: semillas variegadas (Pascualides y Planchuelo, 2007). Este último grupo, tuvo un poder germinativo alto en condiciones de laboratorio; mientras que en el campo, el porcentaje se redujo (Pascualides y Ateca, 2013). El objetivo de este trabajo fue estudiar el efecto de los colores de la cubierta seminal sobre la imbibición, la germinación y los componentes de la semilla. Este tipo de trabajo permitirá profundizar el conocimiento de los factores que influyen en la capacidad de imbibición, germinación y emergencia en el campo de C. juncea.

\section{MATERIALES Y MÉTODOS}

\section{MATERIAL BIOLÓGICO}

Las semillas de $C$. juncea corresponden a la cosecha del año 2012 realizada en Villa del Totoral, Córdoba Argentina (Lat. Sur 3049' y Long. Oeste 634' y $233 \mathrm{msnm}$ ), en un suelo Haplustol éntico, con pH 7,16 y materia orgánica, $1,8 \%$. Las temperaturas promedio anual fueron de $26,5{ }^{\circ} \mathrm{C}$, la máxima y $11,8{ }^{\circ} \mathrm{C}$, la mínima. La Fig. 1 presenta las precipitaciones mensuales desde la siembra (mediados de octubre de 2011), a la cosecha (mayo de 2012) (Bolsa de Cereales de Córdoba, 2012).

Los frutos se cosecharon cuando las semillas quedaron sueltas en su interior, se trillaron a mano y las semillas se clasificaron en dos grupos de colores con la ayuda de un microscopio estereoscópico Zeiss y la carta de colores de Charts (2000). Un grupo correspondió a semillas uniformes, de coloración gris oscura, y el otro a semillas variegadas, con manchas de color castaño (Fig. 2 A y B). Éstas representaron el $20 \%$ del total de semillas obtenidas en las parcelas experimentales.

Crotalaria juncea se caracteriza por presentar semillas endospérmicas, ya que poseen un tejido de varias capas de espesor entre la superficie abaxial de los cotiledones y la superficie interna del espisperma (Esau, 1982; Pascualides, 2004; Corby et al., 2011). 


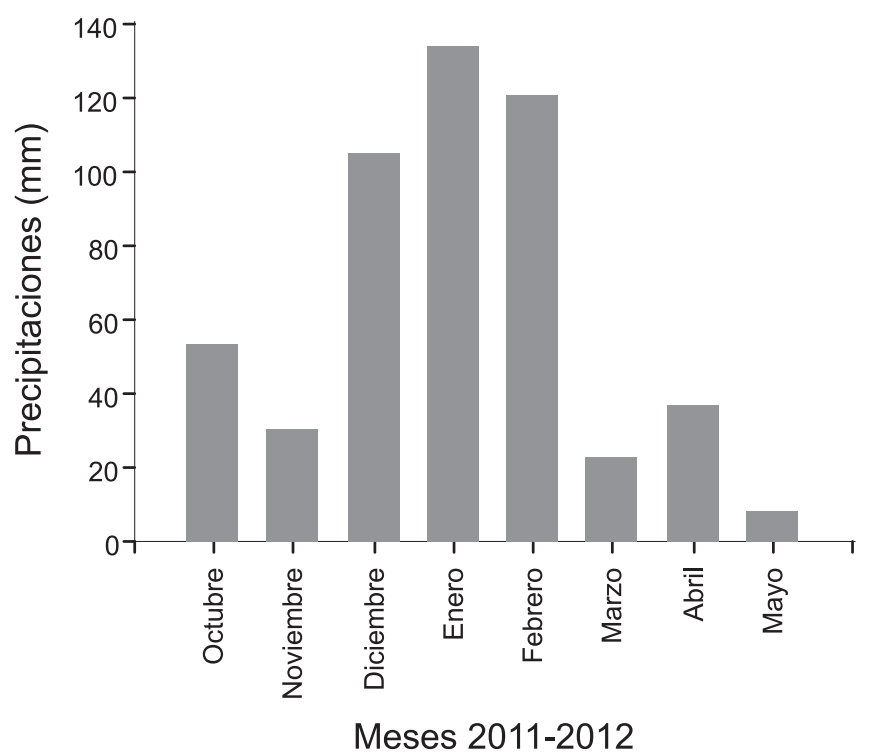

Fig. 1. Precipitaciones mensuales en Villa del Totoral, Córdoba, Argentina en el ciclo del cultivo de C. juncea, 2011-2012.

La Fig. 2 (C y D) muestra los componentes de la semilla (cubierta seminal, embrión curvo y endosperma). Las semillas presentaron un $9 \%$ de humedad (ISTA, 2012).

\section{PROPORCIÓN DE LAS PARTES}

DE LA SEMILLA Y PORCENTAJE DE AGUA RETENIDA POR CADA UNA

Las variables evaluadas fueron: la proporción de la cubierta seminal, del endosperma, del embrión y la cantidad de agua retenida por cada componente. Para ello, se pesaron tres repeticiones de cincuenta semillas uniformes y variegadas (peso total: $\mathrm{Pt}$ ), se colocaron en un beaker con $100 \mathrm{ml}$ de agua destilada a temperatura ambiente durante 12 horas, para facilitar la separación manual de los tres componentes (Ertekin y Kirdar, 2010). Cada parte se ubicó en un envase de papel de aluminio de peso conocido (peso envase: $\mathrm{Pa}$ ); el conjunto fue pesado (peso inicial: $\mathrm{Pi}$ ), y acondicionado en estufa a 60 ${ }^{\circ} \mathrm{C}$ hasta alcanzar peso constante (peso final: Pf). El ensayo presentó un diseño completamente aleatorizado y para la determinación de la proporción en peso de cada parte con respecto al peso total de la semilla se empleó la siguiente fórmula:

$$
\text { Proporción } \%=\frac{P f-P a}{P t} \chi 100
$$

Siendo:

Pt: peso total de la semilla

Pf: peso final

Pa: peso del envase de aluminio

Pi: peso del envase conteniendo un componente de la semilla

Para el cálculo de la cantidad de agua que retiene cada componente de la semilla se utilizó la siguiente fórmula:

$$
\% \text { agua }=\frac{P i-P f}{P i-P a} \chi 100
$$

\section{ESPESOR DE LA CUBIERTA SEMINAL Y DEL ENDOSPERMA}

Se realizaron preparados temporarios de cortes transversales, en la parte media de las semillas previamente embebidas. La cubierta seminal y el endosperma de la semillas de $C$. juncea presentan un patrón estable de tejidos descripto por Miller (1967), Manning y Van Staden (1987) y Pascualides y Planchuelo, (2007) entre otros autores. Este patrón comprende: la cutícula, la capa de macroesclerei- 
das, osteoesclereidas, parénquima colapsado, cutícula del tegumento interno, la capa de aleurona y el parénquima del endosperma, tal como se ilustra en la Fig. 3. Los distintos estratos se midieron con micrómetro de ocular en un microscopio Zeiss monocular. Cada dato se multiplicó por el factor de corrección según la lente objetivo utilizada $(40 \mathrm{X}$ episperma y aleurona, y 10X para el endos- perma). Se registraron imágenes mediante cámara digital Nikon Coolpix S 10.

\section{GERMINACIÓN DE LAS SEMILLAS}

Para la germinación se realizaron 4 repeticiones de 100 semillas para cada color, en rollos de papel de germinación previamente humedecido con agua destilada y colocados en bandejas plásticas de $22 \times 16$
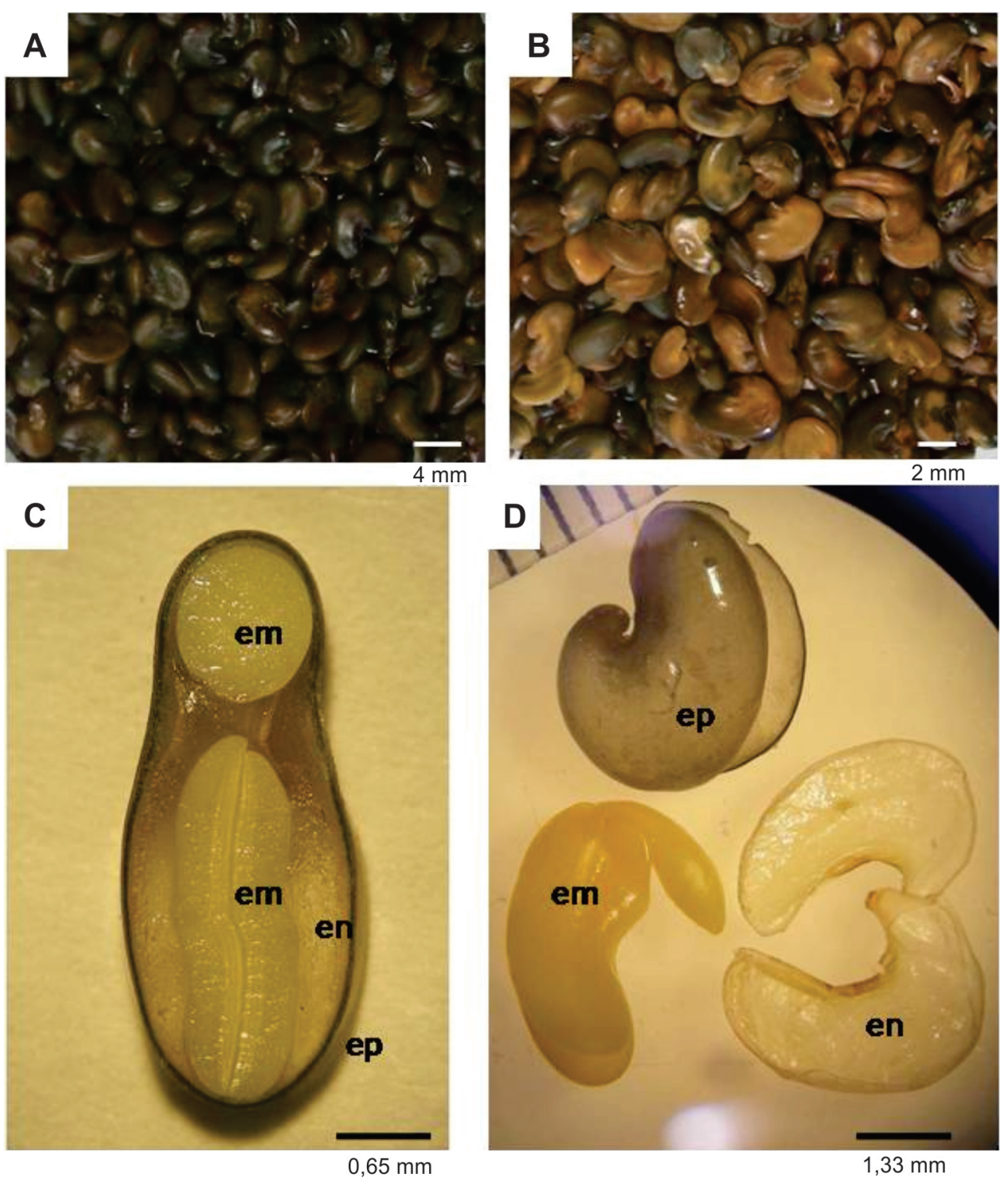

Fig. 2. Grupos de colores y estructura de las semillas de C. juncea. A) Semillas uniformes. B) Semillas variegadas. C) Corte transversal de la semilla. D) Componentes de la semilla. Abreviaturas: ep, episperma o cubierta seminal; em, embrión curvo y en, endosperma. 


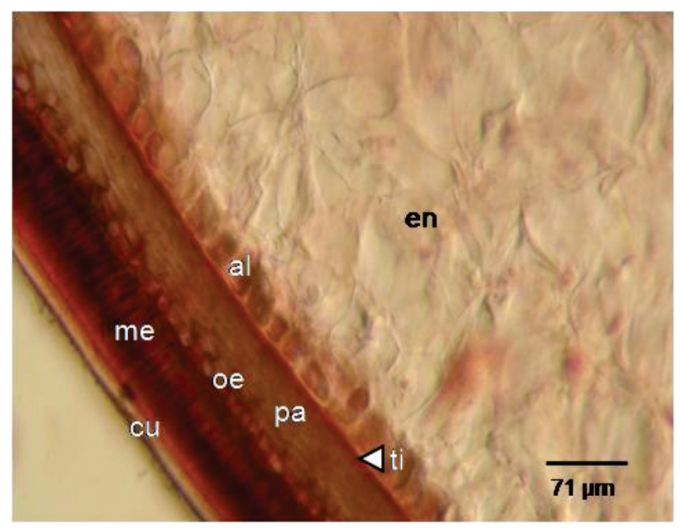

Fig. 3. Histología de la cubierta seminal y endosperma en vista transversal de las semillas C. juncea. Abreviaturas: cu, cutícula; me, macroesclereidas; oe, osteoesclereidas; pa, párénquima; ti, tegumento interno (flecha); al, aleurona; en, parénquima del endosperma.

x $3 \mathrm{~cm}$, cubiertos con bolsas de polietileno. Los rollos fueron colocados en cámara de germinación a temperaturas alternas de 20 ${ }^{\circ} \mathrm{C}$, por 16 horas de oscuridad y $30{ }^{\circ} \mathrm{C}$ con 8 horas de luz (ISTA, 2012). Al cabo de 10 días se determinó el porcentaje de plántulas normales y anormales, semillas duras, frescas y muertas.

\section{EMERGENCIA A CAMPO}

Las semillas se sembraron en parcelas dispuestas en dos hileras de 3 metros de longitud espaciadas a $30 \mathrm{~cm}$ entre sí; la siembra se realizó en forma manual de 2 a $3 \mathrm{~cm}$ de profundidad con una distribución de 8 semillas/m. El experimento presentó un diseño completamente aleatorizado, con tres repeticiones. Además, se sembró perimetralmente para disminuir el efecto de bordura, y durante el ciclo del cultivo se realizaron prácticas culturales de desmalezados manual y riego cuando fue necesario.

\section{ANÁLISIS ESTADÍSTICO}

Se realizó el Análisis de la Varianza y las comparaciones posteriores de las medias con el test de Tukey a un nivel de significancia de 0,01 mediante el Programa InfoStat (Di Rienzo et al., 2012).

\section{RESULTADOS Y DISCUSIÓN}

La Fig. 4 muestra las proporciones de los componentes de los dos colores de la semilla de $C$. juncea. No se observaron diferencias significativas en las proporciones de la cubierta seminal, el endosperma y el embrión entre los dos colores de semillas. La cubierta seminal representó $21 \%$, el endosperma $25 \%$ y el embrión 54\% del total de la semilla; afirmación que se corresponde con las observaciones de Corby et al. (2011), quienes aseveran que en la subfamilia Papilionoideae el embrión representa el 50\% y el endosperma el $22 \%$ del peso total de la semilla (proporción de 1:0,5). Esto se fundamentó en que el embrión contiene las reservas disponibles para la germinación, del mismo modo que se observó en $C$. juncea. En cambio, Liu et al. (2007), encontraron diferencias significativas entre los dos colores de semillas de Cyamopsis tetragonoloba (L.) Taub., para la cubierta seminal (semillas blancas 15,7\% y semillas negras $13,7 \%$ ) y no para el endosperma ni el embrión que representaron el $40 \%$ y $43 \%$ respectivamente. Diferente fue en Gleditsia triacanthos L. donde Ertekin y Kirdar (2010), determinaron mayor proporción de cubierta seminal (68,1-71\%), que de endosperma (27-30,2\%), y muy baja de embrión (1,7-2,1\%); con diferencias significativas entre los tres colores de semillas estudiados, principalmente en la cubierta seminal y el endosperma.

La Tabla 1 muestra los espesores de las capas celulares de la cubierta seminal y el endosperma de los dos colores de semillas de $C$. juncea. El endosperma no reservante favorece la interacción de la semilla con el agua durante los procesos de imbibición y germinación, según lo manifestaron Van Staden et al. (1989), Bewley y Black (1994), ya que este estrato posee una importante capacidad de absorción de agua por la presencia de galactomananos (Corby et al., 2011; Oliva et al., 2010).

Con respecto al porcentaje de agua retenida por cada componente de la semilla de $C$. juncea, se encontraron diferencias altamente significativas entre los dos colores 


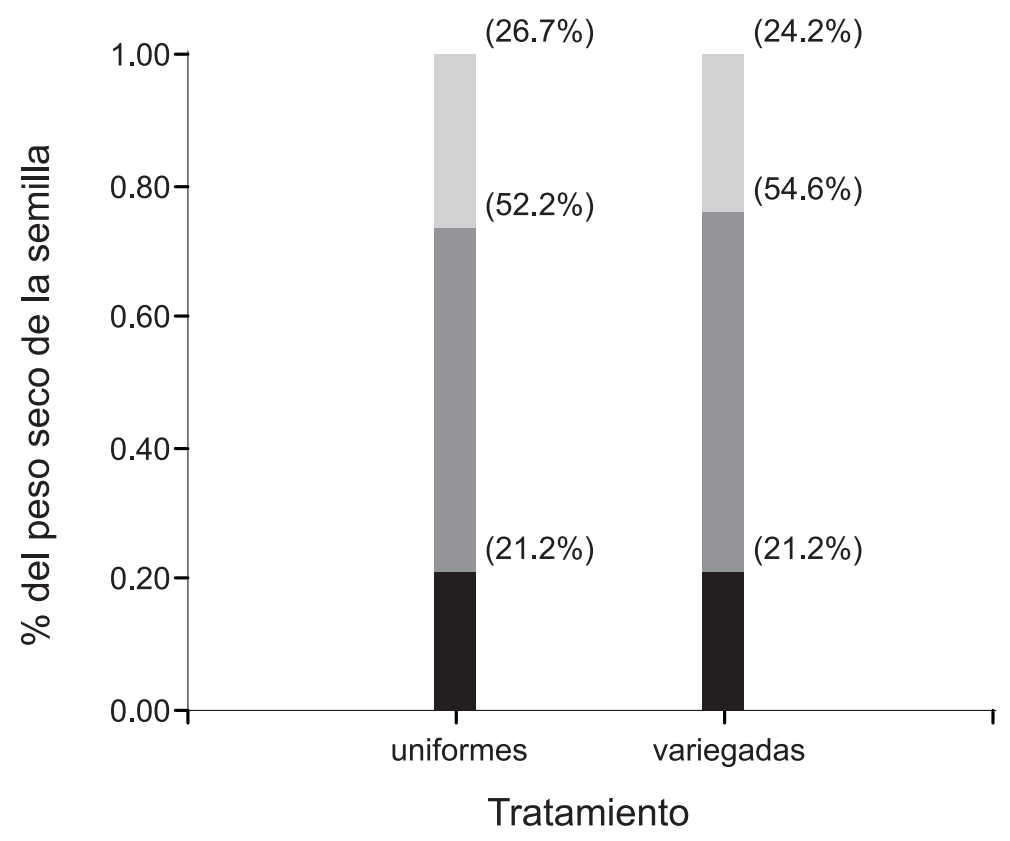

episperma embrión endosperma

Fig. 4. Proporción de cada componente de las semillas uniformes y variegadas de C. juncea.
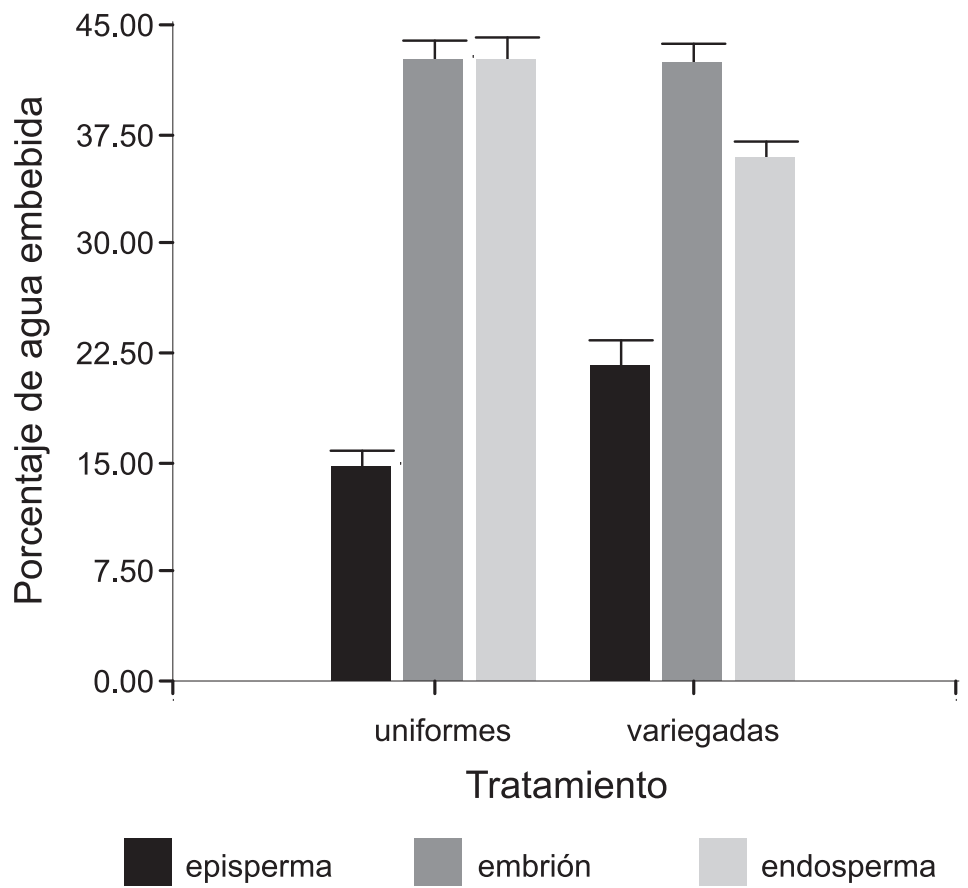

Fig. 5. Porcentaje de agua embebida por el tegumento seminal, el embrión y el endosperma de las semillas uniformes y variegadas de C. juncea. 
para la cubierta seminal $(\mathrm{p}<0,0007)$ y el endosperma ( $\mathrm{p}<0,0001)$ (Fig. 5).

Las semillas variegadas retuvieron mayor porcentaje de agua en la cubierta seminal, la cual presentó mayor espesor que la de las semillas uniformes (Fig.5 y Tabla 1). Del mismo modo, Ertekin y Kirdar (2010), observaron que las semillas de color amarillo de Gleditsia triacanthos, tuvieron mayor captación de agua y mayor espesor de cubierta seminal que las semillas marrones oscuras. En cambio, Liu et al. (2007), encontraron que las semillas claras de Cyamopsis tetragonoloba, embebieron menor porcentaje de agua aunque tuvieron mayor espesor de cubierta seminal que las oscuras.

Las semillas uniformes de C. juncea, absorbieron mayor porcentaje de agua en el endosperma donde la aleurona es de mayor espesor que en las variegadas. La capa de aleurona es uniestrata con células vivas de paredes gruesas (Pascualides y Planchuelo, 2007; Corby et al., 2011). El porcentaje de agua que absorbió el embrión fue similar en los dos colores de semillas.

Las diferencias altamente significativas en el espesor de la cubierta seminal entre las semillas variegadas y uniformes $(\mathrm{p}<0,0001)$, se observó en las capas de las macroesclereidas y del parénquima (Tabla 1 ). Miller (1967), determinó cubiertas seminales de menor espesor en semillas de otras especies del género Crotalaria, tal vez porque no se han incluido la capa de parénquima colapsado y la cutícula del tegumento interno, mencionados por Esau (1982), Fahn (1985) y Pascualides y Planchuelo (2007). Sin embargo, los valores hallados en la cubierta seminal de $C$. juncea observados en este trabajo, se encuentran dentro del intervalo citado por Manning y Van Staden (1987), entre 54 a 124 micrones para el género. Las diferencias altamente significativas $(\mathrm{p}<0,0001)$ en el espesor del endosperma entre los dos colores de semillas se observó en la capa de aleurona, siendo ésta de mayor espesor en las uniformes (Tabla 1). Sin embargo, Miller (1967), registró endosperma de menor espesor en Crotalaria juncea, la diferencia puede deberse al hecho de que el autor no menciona la presencia de la capa de aleurona en su trabajo; la cual fue documentada por Corby et al. (2011), en el endosperma de $C$. ochroleuca G. Don. En las semillas de color uniforme el endosperma total, incluyendo la capa de aleurona, fue el componente que retuvo mayor porcentaje de agua (Fig. 5). Reid y Bewley (1979), encontraron en leguminosas que el endosperma retiene más del 60\% de agua embebida por la semilla y esto disminuye la desecación del embrión si está sujeta a estrés hídrico. Del mismo modo, el endosperma de las semillas de Medicago sativa L. (Hayward, 1953), Ceratonia (Fahn, 1985), Trigonella foenum-graecum L. (Reid y Bewley, 1979), Crotalaria (Corby et al.,

Tabla 1. Espesor de las capas de células que componen la cubierta seminal y el endosperma de semillas de C. juncea.

\begin{tabular}{|c|c|c|c|c|c|c|c|c|}
\hline \multirow[b]{2}{*}{$\begin{array}{l}\text { Coloración } \\
\text { de semilla }\end{array}$} & \multicolumn{6}{|c|}{ Cubierta seminal $(\mu \mathrm{m})$} & \multicolumn{2}{|c|}{ Endosperma $(\mu \mathrm{m})$} \\
\hline & Cutícula & $\begin{array}{c}\text { Macro- } \\
\text { esclereidas }\end{array}$ & $\begin{array}{c}\text { Oste0- } \\
\text { esclereidas }\end{array}$ & Parénquima & $\begin{array}{l}\text { Tegumento } \\
\text { interno }\end{array}$ & $\begin{array}{c}\text { Espesor total } \\
\text { cubierta } \\
\text { seminal }\end{array}$ & Aleurona & $\begin{array}{c}\text { Parénquima } \\
\text { del } \\
\text { endosperma }\end{array}$ \\
\hline Uniformes & $7,63 \pm 0,4 a$ & $43,63 \pm 1 a$ & $16,96 \pm 1,3 a$ & $35,53 \pm 1,6 a$ & $8,04 \pm 0,7 a$ & $111,79 \pm 1 a$ & $36 \pm 1,3 b$ & $633,82 \pm 17,5 a$ \\
\hline Variegadas & $8,3 \pm 0,4 a$ & $49,59 \pm 1 b$ & $14,21 \pm 0,6 a$ & $48,09 \pm 2 b$ & $6,80 \pm 0,3 a$ & $127,04 \pm 0,8 b$ & $28,6 \pm 1 a$ & $572,32 \pm 29,6 a$ \\
\hline
\end{tabular}

Tabla 2. Porcentaje de germinación y emergencia en el campo de semillas de C. juncea.

\begin{tabular}{lc|c|c|c|c|c}
\hline $\begin{array}{l}\text { Coloración } \\
\text { de semilla }\end{array}$ & $\begin{array}{c}\text { Plántulas } \\
\text { normales (\%) }\end{array}$ & $\begin{array}{c}\text { Plántulas } \\
\text { anormales (\%) }\end{array}$ & $\begin{array}{c}\text { Semillas } \\
\text { duras (\%) }\end{array}$ & $\begin{array}{c}\text { Semillas } \\
\text { frescas (\%) }\end{array}$ & $\begin{array}{c}\text { Semillas } \\
\text { muertas (\%) }\end{array}$ & $\begin{array}{c}\text { Emergencia en } \\
\text { el campo (\%) }\end{array}$ \\
\hline Uniformes & $96,5 \pm 1,3 \mathrm{~b}$ & $0,0 \mathrm{a}$ & $2,2 \pm 1,0 \mathrm{a}$ & $0,2 \pm 0,2 \mathrm{a}$ & $1,1 \pm 1,0 \mathrm{a}$ & $80,7 \pm 2,8 \mathrm{~b}$ \\
\hline Variegadas & $80,0 \pm 4,1 \mathrm{a}$ & $0,0 \mathrm{a}$ & $3,7 \pm 0,7 \mathrm{a}$ & $1,0 \pm 0,4 \mathrm{a}$ & $15,3 \pm 3,7 \mathrm{~b}$ & $52.7 \pm 2,5 \mathrm{a}$ \\
\hline
\end{tabular}


2011) y Prosopis, (Oliva et al., 2010), puede intervenir en la regulación de la germinación impidiendo la desecación durante el proceso de imbibición.

En el mismo tiempo de imbibición, las semillas variegadas retuvieron agua en la cubierta seminal; mientras que, las uniformes en el endosperma, tejido más cercano al embrión, esto podría explicar que estas semillas tienen un alto porcentaje de germinación en el laboratorio y un mejor comportamiento en el campo que las semillas variegadas (Tabla 2).

\section{CONCLUSIÓN}

Las semillas uniformes presentaron una ventaja sobre las variegadas, ya que el mayor porcentaje de agua lo retiene el endosperma que está en contacto directo con el embrión y por lo tanto favorece su hidratación completa. En cambio, en las semillas variegadas el mayor porcentaje de agua se encontró en la cubierta seminal; se puede correlacionar esto con el mayor espesor de las macroesclereidas y el parénquima. Estas relaciones pueden explicar que semillas de color uniforme tienen un alto porcentaje de germinación en el laboratorio y un mejor comportamiento en el campo. El color de la cubierta seminal puede tomarse como un indicador para predecir las interacciones entre las estructuras internas de la semilla y el ambiente externo, ya que las capas de células que recubren el embrión influyen en la imbibición y el contenido de humedad, la germinación y la emergencia en el campo.

\section{AGRADECIMIENTOS}

Las autoras agradecen a la Secretaría de Ciencia y Tecnología de la Universidad Nacional de Córdoba por el subsidio otorgado, a Norma Susana Ateca y Raúl Alberto Nobile por las observaciones realizadas en el manuscrito.

\section{BIBLIOGRAFÍA}

Bewley J. D., Black M. 1994. Seeds: physiology of development and germination. Plenum Press, New York.
Buth G. M., Narayan A. 1987. Seed and seed coat anatomy of some members of Crotalaria (Papilionaceae). Journal of the Indian Botanical Society 66: 317-324.

Bolsa de cereales de Córdoba 2012. http:// www.bccba.com.ar

Carreras M. E., Pascualides A. L., Planchuelo A. M. 2001. Comportamiento germinativo de las semillas de Crotalaria incana L. (Leguminosae) en relación a la permeabilidad de la cubierta seminal. AgriScientia 18: 45-50.

Cook C. G., White G. A. 1996. Crotalaria juncea $\mathrm{L}$. A potential multipurpose fiber crop. En Progress in new crops [ed. J. Janick], ASHS Press, Arlington, VA pp. 389-394.

Corby H. D. L., Smith D. L., Sprent J. I. 2011. Size, structure and nitrogen content of seeds of Fabaceae in relation to nodulation. Botanical Journal of the Linnean Society 167: 251-280.

Charts, Munsell Soil Color. (2000). Revised washable edition. Gretag Macbeth, New Windsor, NY.

De Souza F. H. D., Marcos-Filho J. 2001. Seed coat and seed environment relationships in Fabaceae. Revista Brasileira de Botânica 24: 365-375.

Di Rienzo J. A., Casanoves F., Balzarini M. G., González L., Tablada, M., Robledo C. W. 2012. Grupo InfoStat, FCA, Universidad Nacional de Córdoba, Argentina. URL http://www. infostat.com.ar

Egley G. H. 1979. Seed Coat Impermeability and Germination of Showy Crotalaria [Crotalaria spectabilis) Seeds. Weed Science 27: 355-361.

Ertekin M., Kirdar E. 2010. Effects of seed coat color on seed characteristics of honeylocust [Gleditsia triacanthos]. African Journal of Agricultural Research 5: 2434-2438.

Esau K. 1982. Anatomía de las plantas con semilla. Ed. Hemisferio Sur S. A. Buenos Aires. pp. 423-463.

Fahn A. 1985. Las semillas. Anatomía Vegetal. Ed. Pirámides S.A. Madrid. pp. 528-546.

Hayward H. E. 1953. Estructura de las plantas útiles. Leguminosae. Medicago (alfalfa). ACME. Bs. As. pp. 341-371.

ISTA. 2012. International Rules for Seed Testing. The International Seed Testing Association. Switzerland.

Liu W., Peffley E. B., Powell R. J., Auld D. L., Hou A. 2007. Association of seed.coat color with seed water uptake, germination, and seed components in guar $(\mathrm{Cy}$ - 
amopsis tetragonoloba (L.) Taub). Journal of arid Environments 70: 29-38.

Manning J. C., Van Staden J. 1987. The systematic significance of testa anatomy in the Leguminosae - An illustrated survey. South African Journal of Botany 53: 210-230.

Mc Donald Jr. M. B., Vertucci C. W., Roos E. E. 1988. Seed coat regulation of soybean seed imbibition. Crop Science 28: 987-992.

Miller R. H. 1967. Crotalaria seed morphology, anatomy and identification. Technical Bulletin 1373 Agricultural Research Service, pp. 73.

Oliva M., Alfaro C., Palape I. 2010. Evaluation of the technological potential of galactomannan from the endosperm of Prosopis sp. to be used in food industry. AgriScientia 27: 107-113.

Pascualides A. L. 2004. Caracterización morfológica de las semillas de Crotalaria juncea L. y su relación con la calidad fisiológica. Tesis de Maestría en Tecnología de semillas. Facultad de Ciencias Agropecuarias. Universidad Nacional de Córdoba, 104 pp.
Pascualides A. L., Planchuelo A. M. 2007. Seed morphology and imbibition pattern of Crotalaria juncea L. (Fabaceae). Seed Science and Technology 35: 760-764.

Pascualides A. L., Ateca N. 2013. Germinación y vigor de morfotipos de semillas de Crotalaria juncea L. (Fabaceae). Phyton 82: 313-319.

Reid J. S. G., Bewley J. D. 1979. A dual role for the endosperm and its galactomannan reserves in the germinative physiology of fenugreek (Trigonella foenum-graecum L.), an endospermic leguminous seed. Planta 147: 145-150.

Russi L., Cocks P. S., Roberts E. H. 1992. Coat thickness and hard-seededness in some Medicago and Trifolium species. Seed Science Research 2: 243-249.

Saha P. K., Takahashi N. 1981. Seed dormancy and water uptake in Crotalaria sericea Retz. Annals of Botany 47: 423425.

Van Staden J, Manning J. C., Kelly K. M. 1989. Legume Seeds- The Structure: Function Equation. En Advances in Legume Biology. Edited by C. H. Stirton, J. L. Zarucchi 29: 417-450. 